\title{
Ossification of the Posterior Longitudinal Ligament: A Review of Literature
}

\author{
Byung-Wan Choi ${ }^{1}$, Kyung-Jin Song ${ }^{2}$, Han Chang ${ }^{1}$ \\ 'Department of Orthopedic Surgery, Haeundae Paik Hospital, Inje University College of Medicine, Busan, Korea \\ ${ }^{2}$ Department of Orthopedic Surgery, Chonbuk National University Hospital, \\ Chonbuk National University Medical School, Jeonju, Korea
}

Ossification of the posterior longitudinal ligament (OPLL) is most commonly found in men, in the elderly, and in Asian patients. The disease can start with mild or no symptoms, but some patients progress slowly to develop symptoms of myelopathy. An accurate diagnosis through the use plain radiograph, computed tomography, and magnetic resonance imaging findings is very important to monitor the development of symptoms and to make decisions regarding a treatment plan. When symptoms are mild and non-progressive, conservative treatments and periodic observations are good enough, but once symptoms of myelopathy are present and neurologic symptoms are progressive, the treatment of choice is surgery to relieve spinal cord compression. Surgical management of OPLL continues to be controversial. Each surgical technique has some advantages and disadvantages, and the choice of operation should be decided carefully with various considerations. The patient's neurological condition, location and extent of pathology, cervical kyphosis, presence or absence of accompanied instability, and the individual surgeon's experience must be an important factors that should be considered before surgery.

Key Words: Cervical spine, Ossification, Posterior longitudinal ligament

\section{Introduction}

Ossification of the posterior longitudinal ligament (OPLL) is a condition of abnormal calcification of the posterior longitudinal ligament. The most common location is at the cervical spine region. Compression of spinal cord caused by OPLL may lead to neurologic symptoms and in the cases with severe neurologic deficit, surgical treatments are required. However, the exact pathogenesis and natural history of OPLL remain unclear, there is no standard treatment for patients with asymptomatic OPLL, and there is disagreement about the best surgical approach for surgery. In the present review article, we discuss the current devel- opment, natural history, clinical symptoms, classification, radiologic diagnosis, and treatments regarding OPLL.

\section{Epidemiology}

The incidence of OPLL is $2.4 \%$ in the Asian population, and $0.16 \%$ in the non-Asian population [1,2]. OPLL is twice as common in men as it is in women, and symptomatic OPLL usually presents in the 5th to 6th decade of life. OPLL can be associated with other musculoskeletal diseases such as diffuse idiopathic skeletal hyperostosis, ankylosing spondylitis, and other spondyloarthropathy [3-6]. According to Matsunaga et al. [7], schizophrenia patients have a $20 \%$ incidence rate for OPLL.

Received Sep 7, 2011; 1st Revised Sep 23, 2011; Accepted Sep 23, 2011

Corresponding author: Kyung-Jin Song, MD

Department of Orthopedic Surgery, Chonbuk National University Hospital, Chonbuk National University Medical School, 634-18 Geumam-dong, Deokjin-gu, Jeonju 561-712, Korea

Tel: +82-63-250-1770, Fax: +82-63-271-6538, E-mail: kysong@jbnu.ac.kr 


\section{Pathogenesis}

The pathogenesis of OPLL is still unknown. There is some evidence that ligament cells from OPLL patients have osteoblast-like characteristics. In animal models, degeneration or herniation of the nucleus pulposus has been reported as a local factor that initiates OPLL formation. Additional studies on various factors including genetic, hormonal, environmental, and lifestyle have been reported as the cause of pathology and progression of OPLL. However, there is no consensus on this issue [8-12].

OPLL is common in the Asian population and accordingly, genetic factors are considered to be an important factor for the incidence. There have been many studies on collagen genes, including on the human collagen A2 gene (COL11A2). Koga et al. [8] reported that the gene is located at chromosome $6 \mathrm{p}$ nearby human leukocyte antigen region, and seriously involves in development of OPLL. Maeda et al. [9] also reported sex-specific association of COL11A2 haplotype in male OPLL patients. In addition, retinoic $\mathrm{X}$ receptor $\beta$ and collagen 11A2 were also reported to be closely related with OPLL. Bone morphogenic protein (BMP) induces the formation of ectopic bones and cartilage, and is considered to play an important role in the pathogenesis of OPLL. More specifically, BMP-2 stimulates differentiation of ligament cells of OPLL patients, and induces ossification by increasing alkaline phosphatase activity and stimulating DNA and procollagen Type I carboxyl-terminal peptide synthesis [10]. Transforming growth factor (TGF) $\beta$ was also considered to be an important factor for OPLL formation, but Kawaguchi et al. [11] reported that TGF- $\beta 1$ polymorphism is not related to onset of OPLL, but is related to extension of ossification. In addition, insulin-like growth factors, connective tissue growth factors, growth hormone-binding proteins, platelet-derived growth factors and interleukin-7 are considered to be implicated in the development of OPLL [12].

Non-insulin-dependent diabetes mellitus has been suggested to be a risk factor for OPLL. Li et al. [13] reported expression of insulin receptors in OPLL patients, proliferation of rat spinal ligament cells by insulin, and induction of osteogenic differentiation. As OPLL is a disease which increases bone formation in the ligament tissues, studies on the relationship between OPLL and bone mineral density (BMD) have been done and patients with OPLL had higher BMD than the non-OPLL controls [14]. Mechanical stress on spinal ligaments has been investigated as a cause of OPLL development and progression [15]. In studies in which mechanical stress has been applied to the ligament of OPLL patients, prostacyclin synthase levels have increased and accordingly, osteogenic differentiation was induced through the PGI2/cyclic adenosine monophosphate pathway.

Risk factors related to lifestyle, including frequent consumption of pickles, nondaily consumers of rice, family history of myocardial infarction, high body mass index at age 40 , long working hours, and working the night shift are suggested to increase the risk of developing OPLL, whereas frequent intake of chicken or soy products and good sleeping habits reduce the risk of OPLL onset [16,17].

\section{Pathology}

OPLL is formed mainly through enchondral ossification, and part of it develops through membranous ossification. McAfee et al. [18] reported that the pathology of most OPLL cases is composed of a lamellar bone with mature Haversian canals, in addition to fibrous cartilages and woven bones wrapped with calcified cartilage.

Ossification begins at the connection area of lateral limbus of vertebral body and posterior longitudinal ligament, and progresses up and down. OPLL progresses slowly, and shows a wide variety of neuronal degeneration by compressing the spinal cord and nerve roots. However in some cases, good spinal cord function can be preserved without neurologic deficits and with the presence of up to $60 \%$ stenosis in the spinal canal. In accordance with the spinal cord compression, necrosis of the gray matter, decrease in anterior horn cells, and demyelinization of white matter can occure. Autopsy findings of myelopathy patients include necrosis of the gray matter and the white matter. The degree of injury in gray matter, and the degree of cord compression are related to the severity of the myelopathy.

\section{Natural History}

Understanding the natural history of OPLL is important in treating these patients, especially for the establishment of treatment plan for the patients who were accidently diagnosed to have OPLL without symptoms. The natural history of OPLL has not been established, thus a treatment plan for OPLL has not been established. Regarding the natural history of OPLL, Matsunaga et al. [19] reported follow-up 
results of 450 patients for at least 10 years, with a mean follow up period of 17.6 years. Only $17 \%$ of patients without myelopathy at the first visit developed myelopathy during the follow-up period. Based on Kaplan-Meier analysis, the myelopathy-free rate was $71 \%$ after 30 years. Based on this, they suggested that OPLL patients without symptoms of myelopathy do not require prophylactic surgery, and reported that $64 \%$ of patients who had symptoms of myelopathy in the early stages experience aggravation of myelopathy. Upon conducting an analysis of a total 131 OPLL patients who underwent posterior decompression, Chiba et al. [20] reported a $56.5 \%$ of progression rate over 2 years, and is more common in the cases of continuous or mixed type OPLL. However, the study has limitations due to different radiography conditions among institutions and the use of plain radiological findings alone, even though the measurement was adjusted by conducting a thorough computer analysis (Fig. 1).

In a follow-up study on the expression of OPLL symptoms conducted for a total of 156 patients for a mean of 10.8 years [21], the authors of the study reported that both static and dynamic factors are involved in the expression of myelopathy; this expression was more common in the patients who had $60 \%$ or more of compression on spinal canal, and had wide range of motion in cervical spine than other patients.

\section{Clinical Symptoms}

At the early stage, most of OPLL patients do not have symptoms, complain mild pain, discomfort, or numbness in hands. As OPLL grows, symptoms increase in severity due to compression of the spinal cord and nerve roots. The most common symptoms in the early stages of OPLL include dysesthesia and tingling sensation in hands, and clumsiness. With the progression of neurologic deficits, lower extremity symptoms, such as gait disturbance may appear. OPLL patients show symptoms of myelopathy caused by spinal cord compression rather than radicular pain due to nerve roots compression. About $80-85 \%$ of OPLL patients experience a slow progression, but the symptoms become suddenly aggravated or even quadriplegia may appear by mild injuries.

Rigidity of lower extremities is a common physical finding, an increase in deep tendon reflex, and extremity dysesthesia can be shown.

\section{Classification}

As far as classification of OPLL, the method described by Investigation Committee on OPLL of the Japanese Ministry of Public Health and Welfare is most commonly used [1]. According to this method, OPLL is classified into the following: local type, segmental type, continuous type, and mixed type based on plain radiographic findings. The local type of OPLL is observed at the posterior rim of the intervertebral disc; the segmental type is observed at the posterior rim of each vertebral body; the continuous type is observed continuously long over many segments; and the mixed type is a combination of the above types.

\section{Radiological Findings}

Plain radiography is the simplest method to detect OPLL, but it has some limitations especially in the early stages of the disease. In a previous study, we reported low inter- and intraobserver reliability of lateral radiography as a tool for OPLL classification, particularly for continuous type OPLL. The interobserver reliability and intraobserver reliability were only 0.51 and 0.67 , respectively. We emphasized the importance of computed tomography (CT), especially 2D or 3D reconstruction of an image that can be used for accurate diagnosis [22]. The degree of spinal stenosis can be measured using the ratio between the anteroposterior diameter of the spinal canal and the maximum thickness of OPLL mass in the same segment, which is found in the lateral view radiographs or in $\mathrm{CT}$.

Since thoracic spine or thoracolumbar OPLL, and ossification of yellow ligament are commonly accompanied with the OPLL cases, examination on the whole spine is necessary, and CT or magnetic resonance imaging (MRI) is useful in this case because plain radiological exams have limitations. MRI has the advantage of well showing disc herniation if any, and conditions of the spinal cord in a noninvasive method, but has the limitation of showing unclear outline image of bone cortex. CT has the strength of showing accurate the shape and size of any ossified mass. One of the useful findings for diagnosis using MRI is a signal change in the spinal cord. It implies a poor prognosis even after a surgery. According to Sun et al. [23], regardless of hyperintensity on T2-weighted imaging or hypointensity on T1weighted imaging, signal change indicates a severe damage to the spinal cord, and in the case of intramedullary spinal 
cord changes in signal intensity on MRI, anterior approach is recommendable. By comparison, Vedantam et al. [24] reported that $\mathrm{T} 2$-weighted imaging was more useful than T1-weighted imaging for predicting the prognosis after surgery and more specifically, in the cases with a sharp shape, the prognosis was poorer than in other cases. For cases in which OPLL patients consider surgical treatments, dural ossification is one of the important radiological findings. When patients with dural ossification undergo decompression surgery using anterior approach, risk of developing complications such as development of another neurologic symptom or central spinal fluid (CSF) leakage is high. Mizuno et al. [25] reported that CT was the most useful tool in diagnosing dural ossification, which was shown a high with the non-segmental type. Hida et al. [26] classified dural defect findings into 2 types through CT images: double layer sign and single layer sign. Double layer sign was defined as anterior and posterior rims of hyper-dense ossification separated by a central hypodense mass. Ten out of 12 patients with double layer signs showed dural defects, while only one out of 9 patients with single layer signs showed the dural defect. Epstein [27] applied this classification to her patients and demonstrated the same results. In a study of 197 patients who underwent anterior decompression and fusion, Min et al. [28] confirmed that $20(52.6 \%)$ out of 38 cases with the double layer sign had a dural defect, while 3 (3.6\%) out of 22 cases with the single layer sign had a dural defect. They added when the hypodense mass is thicker, the risk of dural defect is high.

Studies on the determination of prognosis based on radiological findings have been conducted. Yagi et al. [29] demonstrated that a positive correlation between postopera- tive expansion of the high signal intensity area of the spinal cord and poor neurological outcomes of patients with cervical OPLL. A risk factor for the expansion of the high signal intensity area was spinal instability.

Matsuyama et al. [30] categorized cross-sectional shapes of spine at the maximal compression area into boomerang, teardrop, and triangular shapes. Among the shapes, the teardrop shape showed the best post-operative recovery, whereas the triangular shape showed the poorest prognosis due to the least expansion after surgery among other shapes.

\section{Management}

As symptomatic treatments, pain medication, topical agents, anti-inflammatory drugs, antidepressants, anticonvulsants, non-steroidal anti-inflammatory drugs and opioid can be applied, and bed rest and assist devices, such as a brace, are recommended for local stabilization. However, once the symptoms of myelopathy, such as gait disturbance and disorders of fine motor movement in the hand develop, appropriate recovery is not expected with conservative treatments.

Surgical approach can be selected based on the degree of myelopathy, the number of involved segments, the location of the primary pathology, the sagittal balance of cervical spine and surgeon's experiences.

\section{Anterior Approach}

After directly removing OPLL in anterior aspect, or floating the OPLL mass from the surrounding bone tissues, the involved segments are fused. When the pathologic focus is
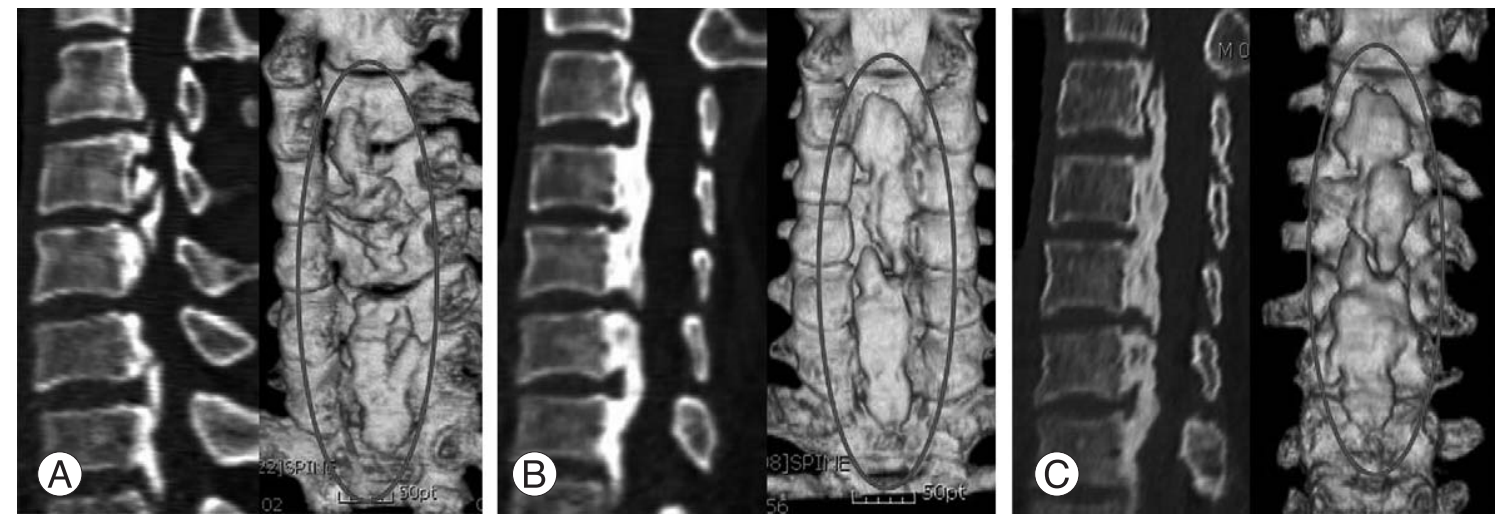

Fig. 1. (A) The sagittal computerized tomography scan of 66-year-old male patient showed mixed type ossification of the posterior longitudinal ligament (OPLL). (B) Follow up copmuted tomography scan of two years after laminoplasty. OPLL mass grew the most 2 years after surgery. (C) Five years after surgery, there showed mild increasement of the OPLL mass than post-operative 2 years finding, but the difference was minimal compared with first 2 years. 
localized below the 3rd-4th cervical vertebrae, a direct anterior approach to the pathology can lead to a good result, but the risk of spinal cord injury is high and in the case that OPLL is not separated from the dura mater, there is a risk of developing complications such as a dural tear or CSF leakage $[31,32]$.

In general, the anterior approach is recommended with the presences of $60 \%$ or more of OPLL occupancy, sharp OPLL shape, and cervical kyphosis, but in the cases of the multisegmental lesion, $\mathrm{C} 2$ and $\mathrm{T} 1$ areas involvement are technically difficult to be performed [33-35]. Surgical methods of anterior approach include anterior cervical disectomy with fusion (ACDF), anterior cervical corpectomy with fusion, open-window corpectomy and anterior decompression via a transvertebral approach, and a $51 \%$ to $71.7 \%$ of improvement rate has been reported (Fig. 2). Koyanagi et al. [36] reported that $60 \%$ or more of OPLL patients showed accompanied disc protrusion at the maximal compression area, and in case of segmental-type OPLL, the rate increased up to $81 \%$. Eventually, ACDF is the useful method of treatment for these patients, and the ratio of postoperative recovery reached $51 \%$ to $63.2 \%$. Onari et al. [33] reported improvements in the 24 out of the total 30 OPLL patients who underwent the anterior fusion without decompression. This method was effective for segmental or local types rather than for continuous type or mixed type. This implies that dynamic factors play an important role in the expression of cervical symptoms in cervical OPLL patients. According to a recent literature review on surgical treatments conducted by Smith et al. [37], even though the anterior decompression shows excellent recovery of neurologic deficits by direct decompression on the pathology, there is a soft tissue problem. In addition, the posterior decompression is useful for elderly patients, but cervical pain still remains and recovery of neurologic deficits is slower than anterior decompression. Song et al. [38] reported 9 cases of OPLL development out of 26 cervicomyelopathy patients, and a $92.3 \%$ of symptoms improvement using anterior decompression and intervertebral fusion.

\section{Posterior Approach}

Posterior approach is a relatively safe method and indirect decompression in the cases of severe spinal cord compression over 3 or more of segments expecting posterior migration effects, and this approach includes laminectomy, laminectomy and fusion, and laminoplasty (Fig. 3). Laminectomy is the simplest method of decompressing the spinal cord at the posterior aspect, but this may result in post-operative instability and kyphotic deformity. However, deformity itself does not have an affect on neurologic results [39].

Anderson et al. [40] found that laminectomy with fusion decreases the risk of postoperative kyphotic deformity and spinal instability compared with laminectomy alone, but functional improvement is similar to laminectomy or laminoplasty. During the posterior decompression, laminectomy on $\mathrm{C} 2$ or $\mathrm{C} 7$ should be carefully conducted to avoid the risk of causing a swan neck deformity. Additionally, when the both sides of facet joint is removed by $50 \%$ or more, combining cervical fusion with posterior decompression should be considered to avoid instability. The $\mathrm{C} 5$ root
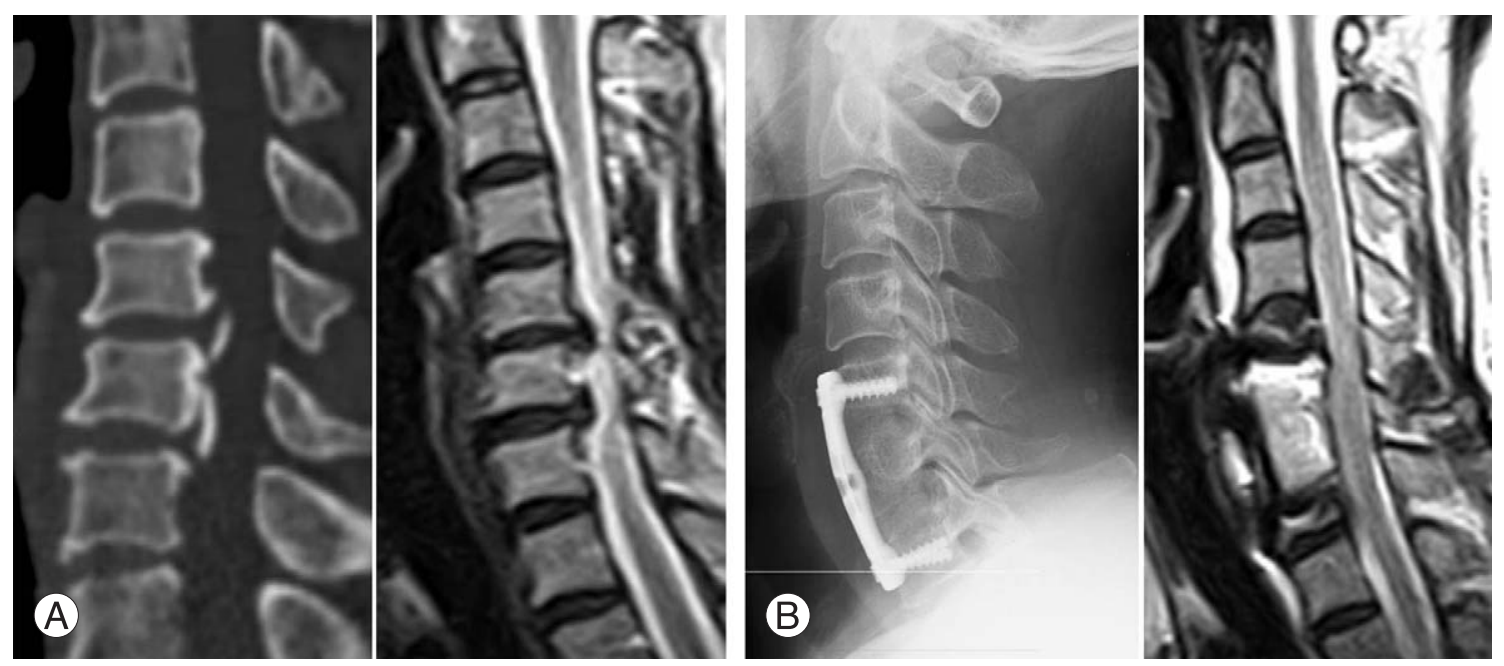

Fig. 2. (A) Preoperative computed tomography and magnetic resonance imaging. (B) After C6 corpectomy and fusion using autogenous strut iliac graft, there showed complete decompression and recovery of subarachnoid space. 

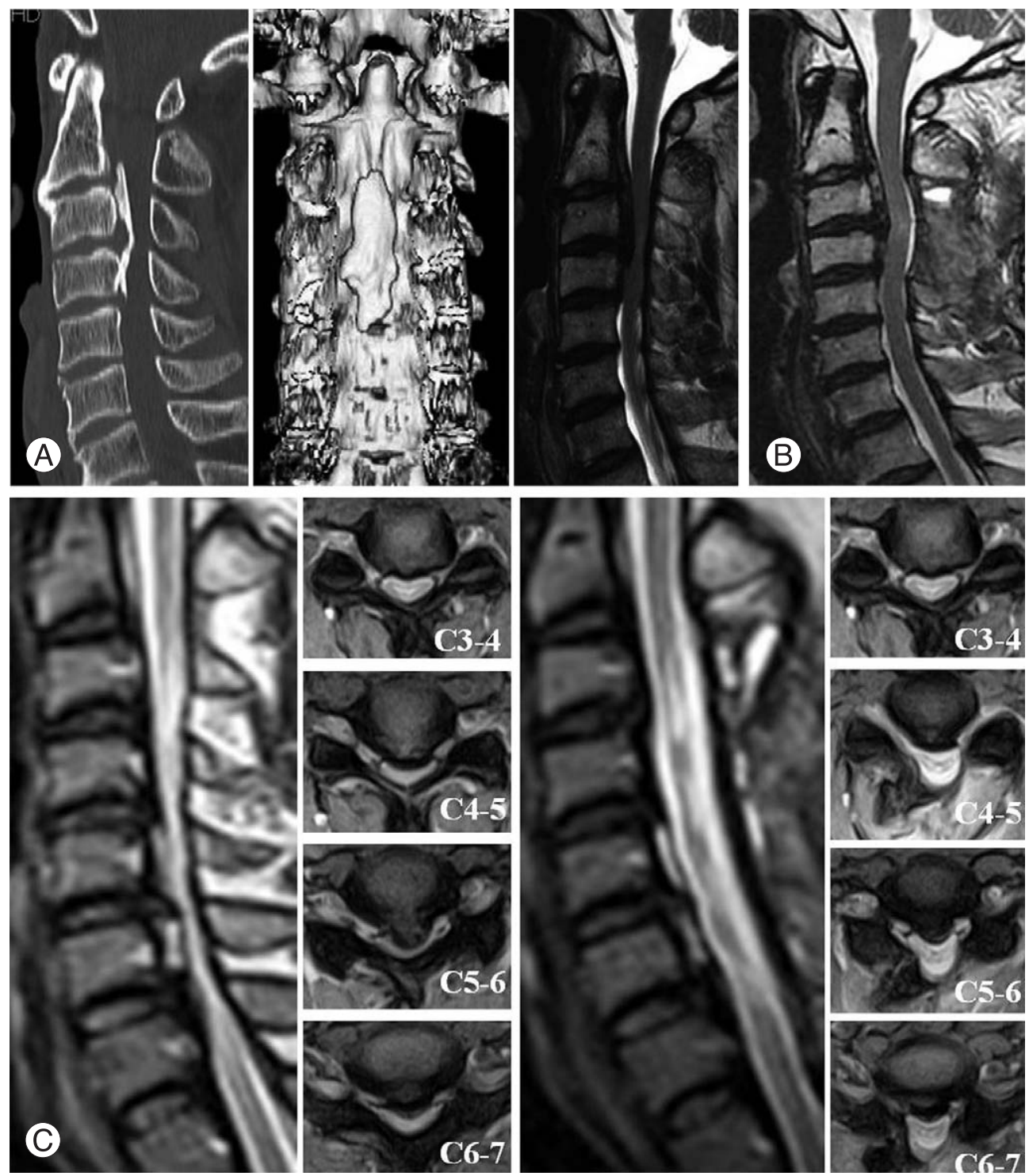

Fig. 3. (A) Preoperative computed tomography and magnetic resonance imaging (MRI). (B) Follow up MRI after posterior en block laminectomy of C3 and C4. (C) Preoperative MRI and follow up MRI. This 56-year-old male patient was performed open door laminoplasty.

palsy developing after the posterior laminectomy and fixation is related with the increase of cervical lordosis, and its major pathogenic mechanism is known as a tethering effect [41,42].

Laminoplasty is a useful method for decompressing the spinal canal posteriorly. Compared with laminectomy, this method has the advantage of reducing kyphotic deformity, which may develop after surgery, and postoperative neurological deficits according to scar tissue formation. There are 2 methods in laminoplasty: open-door and double door methods. Limitation of laminoplasty include axial neck pain, decrease in the cervical range of motion, risk of OPLL progression, limited effects in the cases of severe kyphotic deformity and large OPLL. Especially in case of the opendoor laminoplasty, limited access to hinged side, and risk of reclosure are part of known limitations [43-45]. Various modifications, such as inserting a spacer using an autogenous bone from a spinous process or hydroxyapatite [43], or fixing a miniplate [44] have been introduced to avoid reclosure of the open-door laminoplasty. Hirabayashi et al. [45] compared expansion degree of spinal canal and inclination angle of lamina between the open-door and double-door laminoplasties; wider expansion was obtained from the open-door laminoplasty than the double-door laminoplasty, and inclination angle of lamina significantly increased in case of double-door laminoplasty. The authors suggested 
that open-door laminoplasty can be applied to cervical spondylotic myelopathy (CSM) having unilateral radiculopathy, large prominence of OPLL, and a case double-door laminoplasty cannot be used due to a tiny spinous process. In addition, the authors recommended the open-door laminoplasty for ordinary CSM, for mild OPLL, and for CSM having bilateral nerve root stenosis. According to long-term follow-up results after conducting laminoplasty, a recovery ranging from $47.9 \%$ to $63.1 \%$ has been reported. Factors influencing results of surgical treatment include duration of myelopathy [46], age [47], pre-operative degree of kyphosis, $60 \%$ or more of spinal canal compression, and hill-shape ossification [48]. Poor prognoses have been reported in cases of long symptom duration, old age, severe pre-operative symptoms and traumatic myelopathy.

There are contradictory reports on the degree of OPLL progression and post-operative changes in cervical motion that still inspire controversy. Up to $32 \%$ of cases were reported to have a post-operative decrease in cervical range of motion, but this symptom was reported to not be related with post-operative cervical pain, and no progression was observed 18 months after surgery [49].

Recently, Sakai et al. [50] reported at least 5 years follow-up result of myelopathic patients with OPLL, which was treated by an anterior or posterior approach. In the case of anterior approach, more cases of complication developed that were reported compared with posterior approach, but was more effective for symptoms improvement and was especially useful for the cases of severe spinal canal compression and kyphotic deformity.

According to the multi-center 2-year follow-up study on post-operative OPLL progression conducted by Chiba et al. [51], 56.5\% of patients who underwent the posterior approach experienced the re-growing of the OPLL size. In particular, the size easily increased in the cases of patient 60 years of age or less in age, while the segmental type cases showed less numbers of re-growing compared with other types. Additionally, 70-73\% of posterior decompression cases, and 36-64\% of anterior decompression cases were reported to show progressive increase of OPLL at 10-year follow-up results.

\section{Anterior and Posterior Approach, Combined Approach}

For some cases having localized severe anterior compression accompanied with cervical spinal canal stenosis, the anterior and posterior combined operation can be used. Posterior laminoplasty leads to posterior migration of compressed spinal cord, and finally one is able to conduct anterior decompression more safely.

There are few articles on the combined approach. Thirteen years ago, Epstein [52] confirmed usefulness of the anteroposterior decompression and fusion with 22 patients. Epstein's combined approach was a considerably complicated surgery consuming a mean of 9.8 hours operation time with 3.5 units of blood loss, but the clinical results were satisfactory. According to another report on 65 patients [53], pathology could be removed directly through the combined anterior and posterior fusion, and a maximum stability was obtained immediately after surgery. In addition, grafted bone fracture or displacement was not found when a dynamic plate was used in the anterior fusion, but a high possibility of fusion failure was suggested when the plate was not used or a constrained or semi-constrained plate was used. Accordingly, careful selection of plates and the anterior and posterior approach for multi-segment fusion were recommended. In addition, Song et al. [54] reported satisfactory results of the anterior and posterior approach with fewer complications in the case of myelopathy with kyphotic deformity compared with the results that only the anterior fusion was conducted. For the cases requiring multi-segment anterior decompression, the anterior decompression and fusion with cage only with posterior augmentation with instrumented fusion is considered to be a useful method of reducing the risk of soft tissue damage or graft related complications, and prevents progression of kyphosis in the mid to long-term follow-up. However, to establish applications and usefulness of the anterior and posterior combined approach in OPLL patients, further evaluation of more clinical cases and long-term follow up are needed.

\section{Conclusions}

OPLL is one of the more common factors causing myelopathy in Asian people. Etiology of OPLL is still unknown but genetic, hormonal, environmental and lifestyle factors have been considered to be related with development of OPLL. Radiological analysis of plain radiograph, CT and MRI is essential, and pre-operative meticulous evaluation on maximum compression area of the spinal cord, dural ossification, and presence of signal changes in the spinal cord is important for establishing treatment plans and 
prognoses of the patients. Selection of surgical method for OPLL is still a highly controversial topic and accordingly, advantage and limitation of each method should be well understood. Up to now, the anterior approach has been known to be beneficial for the recovery of neurologic deficits, but surgical method should be selected carefully with the consideration of neurologic status, location of pathology, type of OPLL, presence or absence of kyphotic deformity, associated spinal deformity or medical illness of the patients, and the individual surgeon's experiences.

\section{REFERENCES}

1. Tsuyama N. Ossification of the posterior longitudinal ligament of the spine. Clin Orthop Relat Res 1984;(184):71-84.

2. Wang MY, Thambuswamy M. Ossification of the posterior longitudinal ligament in non-Asians: demographic, clinical, and radiographic findings in 43 patients. Neurosurg Focus 2011;30:E4.

3. Ehara S, Shimamura T, Nakamura R, Yamazaki K. Paravertebral ligamentous ossification: DISH, OPLL and OLF. Eur J Radiol 1998;27:196-205.

4. Guo Q, Ni B, Yang J, Zhu Z, Yang J. Simultaneous ossification of the posterior longitudinal ligament and ossification of the ligamentum flavum causing upper thoracic myelopathy in DISH: case report and literature review. Eur Spine J 2011;20 Suppl 2:S195-201.

5. Khedr EM, Rashad SM, Hamed SA, El-Zharaa F, Abdalla AK. Neurological complications of ankylosing spondylitis: neurophysiological assessment. Rheumatol Int 2009;29:1031-40.

6. Ramos-Remus C, Russell AS, Gomez-Vargas A, et al. Ossification of the posterior longitudinal ligament in three geographically and genetically different populations of ankylosing spondylitis and other spondyloarthropathies. Ann Rheum Dis 1998;57:429-33.

7. Matsunaga S, Koga H, Kawabata N, et al. Ossification of the posterior longitudinal ligament in dizygotic twins with schizophrenia: a case report. Mod Rheumatol 2008;18:27780.

8. Koga H, Sakou T, Taketomi E, et al. Genetic mapping of ossification of the posterior longitudinal ligament of the spine. Am J Hum Genet 1998;62:1460-7.

9. Maeda S, Koga H, Matsunaga S, et al. Gender-specific haplotype association of collagen alpha2 (XI) gene in ossification of the posterior longitudinal ligament of the spine. J Hum Genet 2001;46:1-4.
10. Kon T, Yamazaki M, Tagawa M, et al. Bone morphogenetic protein-2 stimulates differentiation of cultured spinal ligament cells from patients with ossification of the posterior longitudinal ligament. Calcif Tissue Int 1997;60:291-6.

11. Kawaguchi Y, Furushima K, Sugimori K, Inoue I, Kimura $\mathrm{T}$. Association between polymorphism of the transforming growth factor-beta1 gene with the radiologic characteristic and ossification of the posterior longitudinal ligament. Spine (Phila Pa 1976) 2003;28:1424-6.

12. Song J, Mizuno J, Hashizume Y, Nakagawa H. Immunohistochemistry of symptomatic hypertrophy of the posterior longitudinal ligament with special reference to ligamentous ossification. Spinal Cord 2006;44:576-81.

13. Li H, Liu D, Zhao CQ, Jiang LS, Dai LY. Insulin potentiates the proliferation and bone morphogenetic protein-2induced osteogenic differentiation of rat spinal ligament cells via extracellular signal-regulated kinase and phosphatidylinositol 3-kinase. Spine (Phila Pa 1976) 2008;33:2394-402.

14. Hirai N, Ikata T, Murase M, Morita T, Katoh S. Bone mineral density of the lumbar spine in patients with ossification of the posterior longitudinal ligament of the cervical spine. J Spinal Disord 1995;8:337-41.

15. Furukawa K. Current topics in pharmacological research on bone metabolism: molecular basis of ectopic bone formation induced by mechanical stress. J Pharmacol Sci 2006;100:201-4.

16. Okamoto K, Kobashi G, Washio M, et al. Dietary habits and risk of ossification of the posterior longitudinal ligaments of the spine (OPLL); findings from a case-control study in Japan. J Bone Miner Metab 2004;22:612-7.

17. Washio M, Kobashi G, Okamoto K, et al. Sleeping habit and other life styles in the prime of life and risk for ossification of the posterior longitudinal ligament of the spine (OPLL): a case-control study in Japan. J Epidemiol 2004;14:168-73.

18. McAfee PC, Regan JJ, Bohlman HH. Cervical cord compression from ossification of the posterior longitudinal ligament in non-orientals. J Bone Joint Surg Br 1987;69:56975 .

19. Matsunaga S, Sakou T, Taketomi E, Komiya S. Clinical course of patients with ossification of the posterior longitudinal ligament: a minimum 10-year cohort study. J Neurosurg 2004;100(3 Suppl Spine):245-8.

20. Chiba K, Kato Y, Tsuzuki N, et al. Computer-assisted measurement of the size of ossification in patients with ossification of the posterior longitudinal ligament in the cervical 
spine. J Orthop Sci 2005;10:451-6.

21. Matsunaga S, Nakamura K, Seichi A, et al. Radiographic predictors for the development of myelopathy in patients with ossification of the posterior longitudinal ligament: a multicenter cohort study. Spine (Phila Pa 1976) 2008;33:2648-50.

22. Chang H, Kong CG, Won HY, Kim JH, Park JB. Inter- and intra-observer variability of a cervical OPLL classification using reconstructed CT images. Clin Orthop Surg 2010;2:8-12.

23. Sun Q, Hu H, Zhang Y, et al. Do intramedullary spinal cord changes in signal intensity on MRI affect surgical opportunity and approach for cervical myelopathy due to ossification of the posterior longitudinal ligament? Eur Spine J 2011;20:1466-73.

24. Vedantam A, Jonathan A, Rajshekhar V. Association of magnetic resonance imaging signal changes and outcome prediction after surgery for cervical spondylotic myelopathy. J Neurosurg Spine 2011 Sep 16 [Epub]. http://dx.doi.org/10.3171/2011.8.SPINE11452.

25. Mizuno J, Nakagawa H, Matsuo N, Song J. Dural ossification associated with cervical ossification of the posterior longitudinal ligament: frequency of dural ossification and comparison of neuroimaging modalities in ability to identify the disease. J Neurosurg Spine 2005;2:425-30.

26. Hida K, Iwasaki Y, Koyanagi I, Abe H. Bone window computed tomography for detection of dural defect associated with cervical ossified posterior longitudinal ligament. Neurol Med Chir (Tokyo) 1997;37:173-5.

27. Epstein NE. Identification of ossification of the posterior longitudinal ligament extending through the dura on preoperative computed tomographic examinations of the cervical spine. Spine (Phila Pa 1976) 2001;26:182-6.

28. Min JH, Jang JS, Lee SH. Significance of the double-layer and single-layer signs in the ossification of the posterior longitudinal ligament of the cervical spine. J Neurosurg Spine 2007;6:309-12.

29. Yagi M, Ninomiya K, Kihara M, Horiuchi Y. Long-term surgical outcome and risk factors in patients with cervical myelopathy and a change in signal intensity of intramedullary spinal cord on magnetic resonance imaging. J Neurosurg Spine 2010;12:59-65.

30. Matsuyama Y, Kawakami N, Yanase M, et al. Cervical myelopathy due to OPLL: clinical evaluation by MRI and intraoperative spinal sonography. J Spinal Disord Tech 2004; 17:401-4.

31. Cardoso MJ, Koski TR, Ganju A, Liu JC. Approach-related complications after decompression for cervical ossification of the posterior longitudinal ligament. Neurosurg Focus 2011;30:E12.

32. Mazur M, Jost GF, Schmidt MH, Bisson EF. Management of cerebrospinal fluid leaks after anterior decompression for ossification of the posterior longitudinal ligament: a review of the literature. Neurosurg Focus 2011;30:E13.

33. Onari K, Akiyama N, Kondo S, Toguchi A, Mihara H, Tsuchiya T. Long-term follow-up results of anterior interbody fusion applied for cervical myelopathy due to ossification of the posterior longitudinal ligament. Spine (Phila Pa 1976) 2001;26:488-93.

34. Iwasaki M, Okuda S, Miyauchi A, et al. Surgical strategy for cervical myelopathy due to ossification of the posterior longitudinal ligament. Part 1: Clinical results and limitations of laminoplasty. Spine (Phila Pa 1976) 2007;32:64753.

35. Sugrue PA, McClendon J Jr, Halpin RJ, Liu JC, Koski TR, Ganju A. Surgical management of cervical ossification of the posterior longitudinal ligament: natural history and the role of surgical decompression and stabilization. Neurosurg Focus 2011;30:E3.

36. Koyanagi I, Iwasaki Y, Hida K, Imamura H, Abe H. Magnetic resonance imaging findings in ossification of the posterior longitudinal ligament of the cervical spine. J Neurosurg 1998;88:247-54.

37. Smith ZA, Buchanan CC, Raphael D, Khoo LT. Ossification of the posterior longitudinal ligament: pathogenesis, management, and current surgical approaches. A review. Neurosurg Focus 2011;30:E10.

38. Song KJ, Choi BW, Park HJ. Anterior cervical decompression and fusion for the treatment of cervical spondylotic myelopathy. J Korean Orthop Assoc 2002;37:787-94.

39. Kato Y, Iwasaki M, Fuji T, Yonenobu K, Ochi T. Longterm follow-up results of laminectomy for cervical myelopathy caused by ossification of the posterior longitudinal ligament. J Neurosurg 1998;89:217-23.

40. Anderson PA, Matz PG, Groff MW, et al. Laminectomy and fusion for the treatment of cervical degenerative myelopathy. J Neurosurg Spine 2009;11:150-6.

41. Chen Y, Chen D, Wang X, Guo Y, He Z. C5 palsy after laminectomy and posterior cervical fixation for ossification of posterior longitudinal ligament. J Spinal Disord Tech 2007;20:533-5.

42. Chen Y, Guo Y, Chen D, Wang X, Lu X, Yuan W. Longterm outcome of laminectomy and instrumented fusion for cervical ossification of the posterior longitudinal ligament. 
Int Orthop 2009;33:1075-80.

43. Takami T, Ohata K, Goto T, et al. Lift-up laminoplasty for myelopathy caused by ossification of the posterior longitudinal ligament of the cervical spine. Neurol India 2004;52:59-63.

44. Deutsch H, Mummaneni PV, Rodts GE, Haid RW. Posterior cervical laminoplasty using a new plating system: technical note. J Spinal Disord Tech 2004;17:317-20.

45. Hirabayashi S, Yamada H, Motosuneya T, et al. Comparison of enlargement of the spinal canal after cervical laminoplasty: open-door type and double-door type. Eur Spine J 2010;19:1690-4.

46. Iwasaki M, Kawaguchi Y, Kimura T, Yonenobu K. Longterm results of expansive laminoplasty for ossification of the posterior longitudinal ligament of the cervical spine: more than 10 years follow up. J Neurosurg 2002;96(2 Suppl):180-9.

47. Fujimura Y, Nishi Y, Chiba K, Nakamura M, Hirabayashi $\mathrm{K}$. Multiple regression analysis of the factors influencing the results of expansive open-door laminoplasty for cervical myelopathy due to ossification of the posterior longitudinal ligament. Arch Orthop Trauma Surg 1998;117:471-4.

48. Chiba K, Ogawa Y, Ishii K, et al. Long-term results of expansive open-door laminoplasty for cervical myelopathy: average 14-year follow-up study. Spine (Phila Pa 1976) 2006;31:2998-3005.

49. Hyun SJ, Rhim SC, Roh SW, Kang SH, Riew KD. The time course of range of motion loss after cervical laminoplasty: a prospective study with minimum two-year followup. Spine (Phila Pa 1976) 2009;34:1134-9.

50. Sakai K, Okawa A, Takahashi M, et al. 5-year follow-up evaluation of surgical treatment for cervical myelopathy caused by ossification of the posterior longitudinal ligament: a prospective comparative study of anterior decompression and fusion with floating method versus laminoplasty. Spine (Phila Pa 1976) 2011 May 2 [Epub]. http: //dx.doi.org/10.1097/BRS.0b013e31821f4a51.

51. Chiba K, Yamamoto I, Hirabayashi H, et al. Multicenter study investigating the postoperative progression of ossification of the posterior longitudinal ligament in the cervical spine: a new computer-assisted measurement. J Neurosurg Spine 2005;3:17-23.

52. Epstein NE. Circumferential surgery for the management of cervical ossification of the posterior longitudinal ligament. J Spinal Disord 1998;11:200-7.

53. Epstein N. Anterior approaches to cervical spondylosis and ossification of the posterior longitudinal ligament: review of operative technique and assessment of 65 multilevel circumferential procedures. Surg Neurol 2001;55:313-24.

54. Song KJ, Johnson JS, Choi BR, Wang JC, Lee KB. Anterior fusion alone compared with combined anterior and posterior fusion for the treatment of degenerative cervical kyphosis. J Bone Joint Surg Br 2010;92:1548-52. 